Pacific Journal of Mathematics

ON THE PROXIMINALITY OF STONE-WEIERSTRASS 


\title{
ON THE PROXIMINALITY OF STONE-WEIERSTRASS SUBSPACES
}

\author{
JAROSLAV MACH
}

\begin{abstract}
Let $S$ be a compact Hausdorff space, $X$ a Banach space, $C(S, X)$ the Banach space of all continuous $X$-valued functions on $S$ equipped with the supremum norm. In this paper a necessary and sufficient condition on $X$ for every StoneWeierstrass subspace of $C(S, X)$ to be proximinal is established. Furthermore, it is shown that every such subspace is proximinal if $X$ is a dual locally uniformly convex space.
\end{abstract}

Introduction and notations. Let $S$ be a compact Hausdorff space, $X$ a Banach space, $C(S, X)$ the Banach space of all continuous functions on $S$ with values in $X$, equipped with the supremum norm. The purpose of this paper is to study the proximinality of certain subspaces, the so-called Stone-Weierstrass subspaces (SW-subspaces) of $C(S, X)$. This problem has been studied by many authors: Mazur (unpublished, cf., e.g., [11]) proved that every SW-subspace of $C(S, X)$ is proximinal if $X$ is the real line $R$ (a subspace $G$ of a normed linear space $Y$ is called proximinal if every $y \in Y$ possesses an element of best approximation $x_{0}$ in $G$, i.e., if there is an $x_{0} \in G$ such that $\left\|y-x_{0}\right\| \leqq\|y-x\|$ holds for every $\left.x \in G\right)$. Pelczynski [9] and Olech [8] asked for which Banach spaces $X$ every SW-subspace of $C(S, X)$ is proximinal. Olech [8] and Blatter [2] showed that this is true if $X$ is a uniformly convex Banach space and an $L_{1}$-predual space, respectively. It has been shown in [6] that there exists a Banach space $X$ and a compact Hausdorff space $S$ such that $C(S, X)$ has a non-proximinal SW-subspace. Thus, the above mentioned question of characterizing those Banach spaces $X$ for which every SW-subspace is proximinal, arises naturally. Here we give such a characterization. Using a modification of a method due to Olech [8], we show further that if $X$ is a locally uniformly convex space such that every compact subset of $X$ has a Chebychev center (a point $x_{0}$ is called a Chebychev center of a bounded set $F$ if $x_{0}$ is the center of a "smallest" ball containing $F$ ) then every SW-subspace of $C(S, X)$ is proximinal. Every dual space, e.g., has the latter property [3].

We use the following notations. $\boldsymbol{R}$ and $\boldsymbol{N}$ will denote the set of all real numbers and the set of all positive integers, respectively. Let $X$ be a Banach space, $x \in X, r>0 . \quad B(x, r)$ will denote the closed ball in $X$ with center $x$ and radius $r$. A set-valued function $\Phi$ from a topological space $S$ into $2^{X}$ is said to be upper Hausdorff semicon- 
tinuous (u.H.s.c.) respectively lower Hausdorff semicontinuous (l.H.s.c.) if for every $s_{0} \in S$ and every $\varepsilon>0$ there is a neighborhood $U$ of $s_{0}$ such that for every $s \in U$ we have

$$
\sup _{x \in \Phi(s)} \operatorname{dist}\left(x, \Phi\left(s_{0}\right)\right) \leqq \varepsilon
$$

respectively

$$
\sup _{x \varepsilon \Phi\left(s_{0}\right)} \operatorname{dist}(x, \Phi(s)) \leqq \varepsilon
$$

(cf. [10], [12]). The function $\Phi$ is Hausdorff continuous (H.c.) if $\Phi$ is both u.H.s.c. and l.H.s.c. $\Phi$ is l.s.c. respectively u.s.c. if $\Phi$ is lower semicontinuous respectively upper semicontinuous in the usual sense [7]. A Banach space $X$ is said to be locally uniformly convex (l.u.c.) if for every $x \in X$ with $\|x\|=1$ and every sequence $\left\{y_{n}\right\} \subset X$ with $\lim \left\|y_{n}\right\| \leqq 1, \lim \left\|x+y_{n}\right\|=2$ implies $\lim \left\|x-y_{n}\right\|=0$. For a Banach space $X, \mathscr{C}(X)$ will denote the class of all nonempty compact subsets of $X$. For a compact Hausdorff space $S, C(S, X)$ will denote the Banach space of all continuous functions $f$ on $S$ with values in $X$ equipped with the norm $\|f\|=\sup _{s \in S}|f(s)|$, where $|\cdot|$ is the norm of $X$. A subspace $V$ of $C(S, X)$ is said to be an SW-subspace if there is a compact Hausdorff space $T$ and a continuous surjection $\varphi: S \rightarrow T$ such that $V$ consists exactly of those elements $f$ of $C(S, X)$ which have the form $f=g \circ \varnothing$ for some $g \in C(T, X)$. Let $\Phi$ be a function from $S$ into $\mathscr{C}(X)$. A function $f \in C(S, X)$ is said to be a best approximation of $\Phi$ in $C(S, X)$ if the number

$$
\operatorname{dist}(f, \Phi)=\sup _{s \in S} \sup _{x \in \Phi(s)}\|x-f(s)\|
$$

is equal to $\inf \operatorname{dist}(g, \Phi)$, where the infimum is taken over all $g \in$ $C(S, X)$. Let $F$ be a bounded subset of $X$. The number

$$
r(F)=\inf _{x \in X} \sup _{y \in F}\|x-y\|
$$

is called the Chebyshev radius of $F$. A point $x_{0} \in X$ is said to be a Chebyshev center of $F$ if $\left\|x_{0}-y\right\| \leqq r(F)$ for all $y \in F$. The set of all Chebyshev centers of $F$ will be denoted by $c(F)$. For a function $\Phi: S \rightarrow \mathscr{C}(X)$ we denote by $r_{\Phi}$ the number $\sup _{s \in S} r(\Phi(s))$. All Banach spaces in this paper are real.

SW-subspaces of $C(S, X)$. We first establish a simple lemma. Since its proof is straightforward, we omit it here.

LEMma 1. Let $\Phi$ be an u.H.s.c. function from a compact Hausdorff space $T$ into $\mathscr{C}(X)$. Then the set $\bigcup_{t \in T} \Phi(t)$ is compact. 
We formulate now the main theorem of this paper.

THEOREM 2. The following conditions on a Banach space $X$ are equivalent:

(i) For every compact Hausdorff space $T$ and for every u.H.s.c function $\Phi: T \rightarrow \mathscr{C}(X)$, the function

$$
\Psi_{\phi}(t)=\bigcap_{x \in \Phi(t)} B\left(x, r_{\phi}\right), \quad t \in T,
$$

has a continuous selection.

(ii) Every u.H.s.c. function $\Phi$ from an arbitrary compact Hausdorff space $T$ into $\mathscr{C}(X)$ has in $C(T, X)$ a best approximation.

(iii) For any compact Hausdorff space $S$, every SW-subspace of $C(S, X)$ is proximinal.

Proof. (i) $\Rightarrow$ (ii). If $f$ is a continuous selection of $\Psi_{\Phi}$, then $\operatorname{dist}(f, \Phi)=r_{\Phi}$. Further, we obviously have

$$
\inf _{g \in C(T: X)} \operatorname{dist}(g, \Phi) \geqq r_{\Phi} .
$$

It follows that $f$ is a best approximation of $\Phi$.

(ii) $\Rightarrow$ (i). It suffices to show that

$$
\inf _{g \in C(T, X)} \operatorname{dist}(g, \Phi)=r_{\Phi} .
$$

Let $r>r_{\Phi}$ be a fixed number. Let $\Psi_{1}: T \rightarrow 2^{X}$ be defined by

$$
\begin{aligned}
\Psi_{1}(t)= & \{x \in X ; \text { there is a neighborhood } U \text { of } t \text { such } \\
& \text { that } \left.\Phi\left(t^{\prime}\right) \subset B(x, r) \text { for all } t^{\prime} \in U\right\} .
\end{aligned}
$$

We show first that $\Psi_{1}(t) \neq \varnothing$ for every $t \in T$. Since $r(\Phi(t)) \leqq r_{\Phi}<$ $r$, there is an $x_{0} \in X$ for which

$$
\Phi(t) \subset B\left(x_{0},\left(r+r_{\Phi}\right) / 2\right)
$$

holds. Since $\Phi$ is u.H.s.c., there is a neighborhood $U$ of $t$ such that

$$
\sup _{y \in \Phi\left(t^{\prime}\right)} \operatorname{dist}(y, \Phi(t))<\left(r-r_{\Phi}\right) / 2
$$

for every $t^{\prime} \in U$. It follows that $\Phi\left(t^{\prime}\right) \subset B\left(x_{0}, r\right)$ for all $t^{\prime} \in U$. Hence $x_{0} \in \Psi_{1}(t)$. For every $t \in T$ the set $\Psi_{1}(t)$ is obviously convex. It follows immediately from the definition of $\Psi_{1}$ that it is 1.s.c. We put now $\Psi_{2}(t)=\operatorname{cl} \Psi_{1}(t), t \in T$. The map $\Psi_{2}$ is still l.s.c. and therefore it has a continuous selection [7]. Denote this continuous selection by $g$. Let us show now that $\operatorname{dist}(g, \Phi) \leqq r$. To see this, let $\varepsilon>0$ and $t \in T$ be given. There is an $x \in \Psi_{1}(t)$ with $\|g(t)-x\|<\varepsilon$. Consequently, 


$$
\Phi(t) \subset B(g(t), r+\varepsilon) .
$$

Since $\varepsilon$ and $t$ has been arbitrary, we have $\operatorname{dist}(g, \Phi) \leqq r$. Since $r>$ $\boldsymbol{r}_{\Phi}$ has been arbitrary, it follows

$$
\inf _{h \in C(T, X)} \operatorname{dist}(h, \Phi) \leqq r_{\Phi} .
$$

Thus, by (1), we have (2).

(ii) $\Rightarrow$ (iii). This has been essentially proved in [8].

(iii) $\Rightarrow$ (ii). Let $\Phi$ be an u.H.s.c. function from $T$ into $\mathscr{C}(X)$. We show that there is a compact Hausdorff space $S$, a continuous surjection $\varphi: S \rightarrow T$ and a function $f \in C(S, X)$ such that if, for some $g \in C(T, X), g \circ \varphi$ is a best approximation of $f$ in the corresponding SW-subspace $V$, then $g$ is a best approximation of $\Phi$.

By Lemma 1 , there is a number $a>0$ such that $\|x\|<a$ for all for all $x \in \Phi(t)$ and all $t \in T$. Choose an arbitrary $z \in X$ such that $\|\boldsymbol{z}\|>a$ holds. Let $R$ be the subset of $X^{T}$ defined by

$$
\begin{gathered}
R=\left\{s \in X^{T} ;\|s(t)\|<a \text { for some } t \in T \text { and } s\left(t^{\prime}\right)=z\right. \\
\text { for all } \left.t^{\prime} \neq t\right\} .
\end{gathered}
$$

Let $\varphi: R \rightarrow T$ be a function which assigns to every $s \in R$ the only $t \in T$ with $\|s(t)\|<a$. We assume $R$ to be equipped with the following topology $\tau$ : For every $s \in R$ the neighborhood base of $s$ consists of all subsets $W_{\varepsilon, U}$ of $R$ which have the form

$$
W_{\varepsilon, U}=\left\{s^{\prime} \in R ; \psi\left(s^{\prime}\right) \in U \text { and }\left\|s^{\prime}\left(\psi\left(s^{\prime}\right)\right)-s(\psi(s))\right\|<\varepsilon\right\},
$$

where $U$ is a neighborhood from a fixed neighborhood base of $\psi(s)$ and $\varepsilon$ is a positive number. Let $S$ be a subset of $R$ consisting of all $s \in R$ for which $s(\psi(s)) \in \Phi(\psi(s))$ holds. We show that $S$ equipped with the relative topology generated by $\tau$ is a compact Hausdorff space. To verify this, let $\left\{N_{\alpha}\right\}_{\alpha \in A}$ be a covering of $S$ by open subsets of $R$. Let $t \in T$. For every $\alpha \in A$ with $\psi^{-1}(t) \cap N_{\alpha} \neq \varnothing$ let $O_{\alpha}=$ $\left\{s(t) ; s \in \psi^{-1}(t) \cap N_{\alpha}\right\}$. Since $\left\{O_{\alpha}\right\}$ is a covering of $\Phi(t)$ by open subsets of $X$, there exists a finite subcovering $\left\{O_{\alpha_{i}(t)}\right\}, i=1, \cdots, n(t)$. We will show now that there exists an $\varepsilon_{t}>0$ and neighborhood $U_{0}$ of $t$ such that we have

$$
\left\{s ; \psi(s) \in U_{0}\right\} \cap\left\{s ; \operatorname{dist}(s(\psi(s)), \Phi(t))<\varepsilon_{t}\right\} \subset \bigcup_{i=1}^{n(t)} N_{\alpha_{i}(t)} .
$$

Suppose that this is not true. Then for every neighborhood $U$ and every $n \in N$ there exists an $s_{U, n}$ with $\psi\left(s_{U, n}\right) \in U$ and $\operatorname{dist}\left(s_{U, n}\left(\psi\left(s_{U, n}\right)\right)\right.$, $\Phi(t))<1 / n$ which is not in the union of all $N_{\alpha_{i}(t)}, i=1, \cdots, n(t)$. It follows from the compactness of $\Phi(t)$ that there is a cluster point $s_{0} \in S$ of the net $\left\{s_{U, n}\right\}$ with $s_{0}(t) \in \Phi(t)$. The point $s_{0}$ cannot be in the 
union of all $N_{\alpha_{i}(t)}, i=1, \cdots, n(t)$, which implies that $s_{0}(t)$ cannot be in the union of all $O_{\alpha_{i}(t)}, i=1, \cdots, n(t)$. A contradiction.

Now, it follows from the assumption that $\Phi$ is u.H.s.c. that there is an open neighborhood $U_{t}$ of $t$ such that for all $t^{\prime} \in U_{t}$ and all $y \in \Phi\left(t^{\prime}\right)$ we have $\operatorname{dist}(y, \Phi(t))<\varepsilon_{t}$. Moreover, $U_{t}$ can be chosen such that $U_{t} \subset U_{0}$. It follows that

$$
\left\{s \in S ; \psi(s) \in U_{t}\right\} \subset \bigcup_{i=1}^{n(t)} N_{\alpha_{i}(t)} .
$$

Construct such a neighborhood $U_{t}$ for every $t \in T$ and choose a finite subcovering $U_{t_{1}}, \cdots, U_{t_{m}}, m \in N$, of $T$. Then the sets $N_{\alpha_{i}\left(t_{j}\right)}, i=1$, $\cdots, n\left(t_{j}\right), j=1, \cdots, m$, are obviously a finite subcovering of $S$.

The restriction $\varphi$ of $\psi$ to $S$ is obviously a continuous surjection from $S$ onto $T$. Let $f: S \rightarrow X$ be defined by $f(s)=s(\varphi(s))$. The function $f$ is obviously continuous. Let $g \circ \varphi$ be a best approximation of $f$ in the corresponding SW-subspace $V$. Then we have

$$
\begin{aligned}
\operatorname{dist}(g, \Phi) & =\|f-g \circ \varphi\|=\inf _{h \in C(T, X)}\|f-h \circ \varphi\| \\
& =\inf _{h \in C(T, X)} \operatorname{dist}(h, \Phi) .
\end{aligned}
$$

Hence $g$ is a best approximation of $\Phi$ in $C(T, X)$. This completes the proof of the theorem.

Let $\Phi$ be an u.H.s.c. function from $S$ into $\mathscr{C}(X)$. We establish now a sufficient condition for the existence of a continuous selection of $\Psi_{\varphi}$.

Definition. A Banach space $X$ is said to have the property (QUCC) if $c(K) \neq \varnothing$ for every $K \in \mathscr{C}(X)$ and if the following is true: Given a set $K \subset \mathscr{C}(X)$, an element $x \in X$ and numbers $r>0, \varepsilon>0$, there is a $\delta>0$ such that for every $y \in X$ there exists an element $z_{y} \in B(x, \varepsilon)$ satisfying

$$
B(x, r+\delta) \cap B(y, r) \cap K \subset B\left(z_{y}, r\right) \cap K .
$$

THEOREM 3. Let $X$ be a Banach space with the property (QUCC), $S$ a compact Hausdorff space, $\Phi: S \rightarrow \mathscr{C}(X)$ an u.H.s.c. map. Then $\Psi_{\Phi}$ has a continuous selection.

Proof. We show that $\Psi_{\Phi}$ is 1.s.c. First, since for all $t \in T$ $c(\Phi(t)) \subset \Psi_{\Phi}(t)$, we have $\Psi_{\Phi}(t) \neq \varnothing$ for every $t \in T$. Let $t \in T, x \in \Psi_{\Phi}(t)$ and $\varepsilon>0$ be given. For $x, K=\mathbf{U}_{t \in T} \Phi(t)$ (which is a compact set by Lemma 1), $r=r_{\Phi}$ and $\varepsilon$ find the corresponding $\delta$. Since $\Phi$ is u.H.s.c., there is a neighborhood $U$ of $t$ with $\Phi\left(t^{\prime}\right) \subset B(x, r+\delta) \cap K$ 
for every $t^{\prime} \in U$. For $t^{\prime} \in U$ let $y \in \Psi_{\Phi}\left(t^{\prime}\right)$. Then $\Phi\left(t^{\prime}\right) \subset B(x, r+\delta) \cap$ $B(y, r) \cap K \subset B\left(z_{y}, r\right) \cap K$. Hence $z_{y} \in B(x, \varepsilon) \cap \Psi_{\Phi}\left(t^{\prime}\right)$. The existence of a continuous selection of $\Psi_{\phi}$ follows then from Michael's selection theorem [7].

The following proposition provides an example of a class of Banach spaces with the property (QUCC). To prove it, we need the following easy lemma which we state without proof.

LEMMA 4. Let $\left\{s_{n}\right\},\left\{t_{n}\right\}$ be two sequences in a Banach space $X$. Let for some $r>0 \lim \left\|s_{n}\right\| \leqq r, \lim \left\|t_{n}\right\| \leqq r$. Let

$$
u_{n}=\lambda_{n} s_{n}+\left(1-\lambda_{n}\right) t_{n}
$$

be such that we have $\beta_{0} \leqq \lambda_{n} \leqq \eta_{0}$ for some $0<\beta_{0}<1,0<\eta_{0}<1$ and every $n \in N$, and such that $\lim \left\|u_{n}\right\| \geqq r$. Then $\lim \left\|\left(s_{n}+t_{n}\right) / 2\right\| \geqq r$ for suitable subsequences.

Proposition 5. Let $X$ be a l. u. c. space such that $c(K) \neq \varnothing$ for every $K \in \mathscr{C}(X)$. Then $X$ has the property (QUCC).

Proof. Assume the contrary. Then there exist positive numbers $\varepsilon$ and $r$, an element $x \in X$ and a compact set $K \subset X$, such that for every $n \in N$ there is a $y_{n} \in X$ and a $w_{n} \in K$ with $\left\|x-w_{n}\right\| \leqq r+1 / n$, $\left\|y_{n}-w_{n}\right\| \leqq r$, and $\left\|z_{n}-w_{n}\right\|>r$, where

$$
z_{n}=\left(1-\varepsilon / 2 a_{n}\right) x+\left(\varepsilon / 2 a_{n}\right) y_{n}
$$

and $a_{n}=\left\|x-y_{n}\right\|$. One can obviously assume $a_{n}>\varepsilon$ for every $n \in$ $N$. Without loss of generality we can further assume that $w_{n}$ converges to some $w_{0} \in K$. It follows that $\left\|x-w_{0}\right\| \leqq r,\left\|y_{n}-w_{0}\right\| \leqq$ $r+\eta_{n},\left\|z_{n}-w_{0}\right\|>r-\eta_{n}$ for every $n \in N$ holds, where $\eta_{n}=\left\|w_{n}-w_{0}\right\|$. For every $n \in N$ denote $t_{0}=x-w_{0}, s_{n}=y_{n}-w_{0}, u_{n}=z_{n}-w_{0}$. Without loss of generality one can now assume that $\lim \left\|s_{n}\right\| \leqq r$ and $\lim \left\|u_{n}\right\| \geqq r$. Thus, by Lemma 4 , we have $\lim \left\|\left(t_{0}+s_{n}\right) / 2\right\| \geqq r$ which, together with $\left\|t_{0}-s_{n}\right\|=a_{n}>\varepsilon, n \in N$, contradicts the local uniform convexity of $X$.

The following corollary is an immediate consequence of Theorems 2 and 3 and Proposition 5.

CoRollary 6. Let $X$ be a dual l.u.c. space. Let $S$ be a compact Hausdorff space. Then every $\mathrm{SW}$-subspace of $C(S, X)$ is proximinal.

Proof. By a result of Garkavi [3], $c(F)$ is nonempty even for every bounded subset of $X$. 
It is an easy consequence of Lindenstrauss' well-known theorem concerning intersection properties of balls in $L_{1}$-predual spaces with centers in a compact set that these spaces also have the property (QUCC). So we have the following result of Blatter [2].

CoROLlaRY 7. Let $X$ be an $L_{1}$-predual space, $S$ a compact Hausdorff space. Then every SW-subspace of $C(S, X)$ is proximinal.

Ward [13] proved that $c(F) \neq \varnothing$ for every bounded subset of $C(S, X)$ if $X$ is a Hilbert space and $S$ is an arbitrary topological space. Amir [1] and Lau [4], independently, improved this result by showing that this is true for every $X$ uniformly convex. We show now that, for compact subsets of $C(S, X)$ with $S$ compact Hausdorff, this still remains true, if $X$ has the property (QUCC).

THEOREM 8. Let $S$ be a compact Hausdorff space, $X$ a Banach space with the property (QUCC). Then $c(K) \neq \varnothing$ for every compact subset $K$ of $C(S, X)$.

\section{Proof. Let}

$$
\Phi(s)=\{x \in X ; x=f(s) \text { for some } f \in K\}, \quad s \in S .
$$

Then $\Phi$ obviously is a H.c. map from $S$ into $\mathscr{C}(X)$. Furthermore, it is easy to show that $r(K) \geqq r_{\phi}$. Hence every continuous selection of $\Psi_{\phi}$ is in $c(K)$. The assertion of the theorem follows then from Theorem 3.

Corollary 9. Let $X$ be a dual l. u. c. space, $S$ a compact Hausdorff space. Then $c(K) \neq \varnothing$ for every compact subset $K$ of $C(S, X)$.

\section{REFERENCES}

1. D. Amir, Chebychev centers and uniform convexity, Pacific J. Math., 77 (1978), 1-6. 2. J. Blatter, Grothendieck spaces in approximation theory, Mem. Amer. Math. Soc., 120 (1972).

3. A. L. Garkavi, The best possible net and the best possible cross-section of a set in a normed space, Amer. Math. Soc. Transl., 39 (1964), 111-132.

4. K. S. Lau, Approximation by continuous vector-valued functions, Studia. Math.. 68 (1980), 291-298.

5. J. Lindenstrauss, Extension of compact operators, Mem. Amer. Math. Soc., 48 (1964).

6. J. Mach, Continuity properties of Chebychev centers, J. Approximation Theory., 29 (1980), 223-230

7. E. Michael, Continuous selections, Ann. of Math., 63 (1956), 361-382.

8. C. Olech, Approximation of set-valued functions by continuous functions, Colloq. Math., 19 (1968), 285-293.

9. A. Pelczynski, Linear extensions, linear averagings and their application to linear 
topological classification of spaces of continuous functions, Dissert. Math., (Rozprawy Math.), 58, Warszawa, 1968.

10. W. Pollul, Topologien auf Mengen von Teilmengen und Stetigkeit von mengenwertigen metrischen Projektionen, Diplomarbeit, Bonn, 1967.

11. Z. Semadeni, Banach spaces of continuous functions, Monografje Matematyczne 55, Warszawa, 1971.

12. I. Singer, The theory of best approximation and functional analysis, Reg. conference ser. appl. math., 13, SIAM, Philadelphia, 1974.

13. J. D. Ward, Chebyschev centers in spaces of continuous functions, Pacific J. Math., 52 (1974), 283-287.

Received December 21, 1979 and in revised form January 22, 1981.

INSTITUt FÜR ANGEWANDTE MathematiK

DER UNIVERSITAT BONN

WEGELERSTR 6

5300 BONN

Federal Republic of Germany

Current address: Department of Mathematics

Texas A \& M University

College Station, TX 77843 


\section{PACIFIC JOURNAL OF MATHEMATICS}

\section{EDITORS}

DONALD BABBITT (Managing Editor)

University of California

Los Angeles, California 90024

\section{Hugo Rossi}

University of Utah

Salt Lake City, UT 84112

C. C. MOore and Arthur AGuS

University of California

Berkeley, CA 94720
J. DugundJI

Department of Mathematics University of Southern California Los Angeles, California 90007

R. FinN and J. Milgram Stanford University Stanford, California 94305

ASSOCIATE EDITORS
R. ARENS
E. F. BECKENBACH
B. H. NeumanN
F. WOLF
K. YOSHIDA

\section{SUPPORTING INSTITUTIONS}

UNIVERSITY OF ARIZONA

UNIVERSITY OF BRITISH COLUMBIA

CALIFORNIA INSTITUTE OF TECHNOLOGY

UNIVERSITY OF CALIFORNIA

MONTANA STATE UNIVERSITY

UNIVERSITY OF NEVADA, RENO

NEW MEXICO STATE UNIVERSITY

OREGON STATE UNIVERSITY
UNIVERSITY OF OREGON

UNIVERSITY OF SOUTHERN CALIFORNIA

STANFORD UNIVERSITY

UNIVERSITY OF HAWAII

UNIVERSITY OF TOKYO

UNIVERSITY OF UTAH

WASHINGTON STATE UNIVERSITY

UNIVERSITY OF WASHINGTON 


\section{Pacific Journal of Mathematics}

Vol. 99, No. $1 \quad$ May, 1982

Mariano Giaquinta, Jindrich Necas, O. John and J. Stará, On the

regularity up to the boundary for second order nonlinear elliptic systems . . 1

Siegfried Graf, Realizing automorphisms of quotients of product $\sigma$-fields . . 19

Alfred Washington Hales and Ernst Gabor Straus, Projective colorings . . . 31

Sandra Hayes, The weak Nullstellensatz for finite-dimensional complex

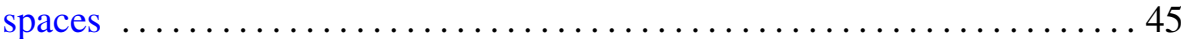

Gerald Norman Hile and Murray Harold Protter, The Cauchy problem

and asymptotic decay for solutions of differential inequalities in Hilbert

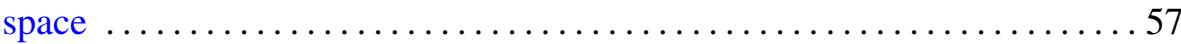

Robert D. Little, Projective space as a branched covering with orientable

branch set ......................................... 89

Jaroslav Mach, On the proximinality of Stone-Weierstrass subspaces . . . . . 997

John C. Morgan, II, On product bases ...................... 105

K. Balakrishna Reddy and P. V. Subrahmanyam, Altman's contractors

and fixed points of multivalued mappings . .................. 127

James Ted Rogers Jr., Decompositions of homogeneous continua . . . . . . . 137

Ahmed Ramzy Sourour, Characterization and order properties of

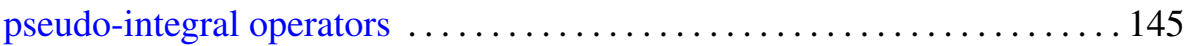

Robert Moffatt Stephenson Jr., Pseudocompact and Stone-Weierstrass

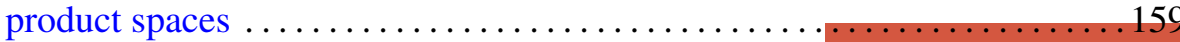

Bruce Stewart Trace, On attaching 3-handles to a 1-connected

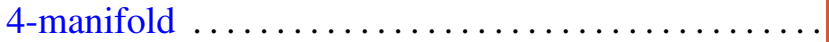

Akihito Uchiyama, The construction of certain BMO functions and the corona problem

Thomas Alva Whitehurst, An application of orthogonal polynomials to random walks ..............................

David J. Winter, Root locologies and idempotents of Lie and nonassociative algebras

William Robin Zame, The classification of uniform algebras on plane domains 